\title{
Activity enhancement of activating transcription factor 3 by restin, a novel melanoma-associated antigen
}

\author{
Junqing $\mathrm{Xu}^{1}$, Yan Xue ${ }^{2}$, Zifan $\mathrm{Lu}^{3}$, Mengqi Wei ${ }^{1}$, Haiyan $\mathrm{Fu}^{3}$, Yong Yang ${ }^{1}$, Fan Lu${ }^{3}$, Yousheng \\ $\mathrm{Wu}^{3^{*}}$ and Yi Huan ${ }^{1^{*}}$
}

\author{
${ }^{1}$ Department of Radiology, Xijing Hospital, Fourth Military Medical University, Xi'an, 710032, China. \\ ${ }^{2}$ Department of Oncology, Xijing Hospital, Fourth Military Medical University, Xi'an, 710032, China. \\ ${ }^{3}$ Department of Biochemistry, Fourth Military Medical University, Xi'an, 710032, China.
}

Accepted 13 December, 2011

\begin{abstract}
Restin, a novel member of the melanoma-associated antigen super family, is cloned from differentiated leukemia cells induced by retinoic acid. Restin may interact with some transcription factors. The present study investigated the effect of restin on activating transcription factor 3 (ATF3), an important protein in numerous physiological situations, using the mammalian two-hybrid system. The results show that ATF3 strongly interacted with restin. To determine the effect of restin on ATF3 activity, an ATF3 reporter plasmid with a luciferase gene was constructed. Hela and NIH3T3 cells transfected with both restin and ATF3 exhibited accelerated ATF3 activity compared with those transfected with only ATF3. This result suggests the role of restin in facilitating ATF3 activity. Restin was also shown to upregulate ATF3 activity by its interaction with the ATF3 DNA binding domain. Overall, these data suggests the involvement of restin in important cellular modulation processes in Hela and NIH3T3 cells by enhancing ATF3 activity.
\end{abstract}

Key words: Restin, MAGE, ATF3, two-hybrid system, ATF3 reporter, luciferase.

\section{INTRODUCTION}

The first melanoma-associated antigen (MAGE) encoding gene was isolated from a melanoma cell. To date, more than thirty members of MAGE have been identified and divided into MAGE-A, B, C, D, E, F, G, H, etc. subfamilies (Chomez et al., 2001). They have also been categorized into two groups, namely, cancer/testis (CT) and non-CT antigens. MAGE-A, B, C, and E are CT antigens. They are expressed in almost all tumors and in normal genital tissues. On the other hand, MAGE-D, F, G, H, and necdin are non-CT antigens and they are mainly expressed in normal tissues including testes, but not in tumor tissues (Francois et al., 2009; Lucas et al., 1998; Reddy et al., 2010). Previous studies have indicated that MAGE proteins contribute to the regulation of cell proliferation and differentiation. MAGE proteins are also closely correlated with neoplasia, wound healing, and

\footnotetext{
*Corresponding author. E-mail: redgem2001@163.com.

Tel/Fax: +862984775421.
}

reproduction (Aprelikova et al., 2009; Francois et al., 2009; Lucas et al., 1998; Reddy et al., 2010).

In our previous study of cell differentiation induced by retinoic acid, restin, a novel MAGE gene was isolated. Restin has been found to encode 229 amino acids (GenBank accession nos. AF143235 and NM_014061) (Zhao et al., 2002; Zhu et al., 2000). Similar to necdin (Maruyama et al., 1991) and MAGE-D1 (Reddy et al., 2010), restin is a non-CT antigen and shares the same high homolog with necdin (Zhao et al., 2002; Zhu et al., 2000). The physical and chemical characteristics of restin are evidently different from those of other MAGE proteins (Zhao et al., 2002; Zhu et al., 2000). CT MAGEs possibly act as accelerators of cell proliferation because they are highly expressed during early stages of tissue wounds (Kirkin et al., 1998). Restin may also be involved in cell proliferation inhibition (Zhao et al., 2002). As a nucleoprotein, we have suspected that restin may fully realize its biological functions via interacting with transcription factors. This hypothesis has been confirmed in our previous studies, which revealed a strong interaction 
of activating transcription factor 3 (ATF3) with restin (Wu et al., 2005; Xu et al., 2006).

However, the domain of ATF3 that interacts with restin is unclear. The biological function of restin on the activity and expression of ATF3 is also not completely understood. The present study aimed to clarify these issues using an ATF3 reporter plasmid pATF/CRE-luc constructed in one of our previous studies (Xu et al., 2006). Restin was found to interact with the ATF3 DNA binding domain (DBD) because the interaction was abolished after the cleavage of this domain. A dose-response promotion of restin on the transcriptional activity of ATF3 was also revealed. Restin was further highlighted as a potential upstream modulator of ATF3.

\section{MATERIALS AND METHODS}

\section{Cell culture}

Hela and NIH3T3 cells were routinely maintained in $10 \%$ fetal calf serum (Sijiqing, China) or Dulbecco's modified Eagle's medium (Promega) as previously described (Freshney, 1994). The cultures were maintained at $37^{\circ} \mathrm{C}$ in a $5 \% \mathrm{CO}_{2}$-air incubator. The cells grew in a confluent monolayer, and were used in the transfection experiments at 80 to $90 \%$ confluence.

\section{Vector construction and preparation}

The construction of pATF/CRE-luc reporter plasmids for ATF3 containing three ATF/CRE binding sites and a luciferase gene had been reported in earlier studies (Xu et al., 2006). The plasmids $\mathrm{pACT} / \mathrm{ATF} 3, \mathrm{pACT} / \mathrm{restin}$, and $\mathrm{pBind} / \mathrm{restin}$ were previously constructed in our laboratory. The cDNA of the ATF3 gene coding sequence (CDS) (546 kb) was cloned into the BamHI/Mul sites of the multiple cloning site (MCS) of the PACT plasmid. While the cDNA of the restin gene CDS $(654 \mathrm{~kb})$ was cloned into the $\mathrm{BamHI} / \mathrm{Mul}$ sites of the pBind and pACT plasmid MCSs. Vectors containing the DBD and transcriptional activation domain (AD) of the ATF3 gene cloned into the BamHI/Mul sites of the PACT plasmid MCS were also constructed. The specific primers were designed as follows: AD-forward: 5'-GCAGGATCCTGATGCTTCAACACCC AGG-3', AD-reverse: 3'-TCGACGCGTCTCGGCTTTTGTGATG-5', BD-forward: 5'-GCAGGATCCGAGGTAGCCCCTG AA-3', BD-reverse: 3'-TCGACGCGTGCTTAGCTCTGCAATGTTCC-5'.

\section{Transient transfection and two-hybrid system between restin and ATF3}

A mammalian two-hybrid system kit containing the plasmids $p B I N D$, pACT, pG5-luc, pBIND-id, and pACT-Myod, as well as AMV reverse transcriptase, RNasin, Transfast transfection reagent, and dualluciferase reporter assay system were obtained from Promega Co. The two-hybrid system was used to determine the domain of ATF3 that interacts with restin. The endonuclease-cutting site in the domain was used as a restriction to assess the boundaries of the completive domain. The interactions of restin with ATF3 and the domain in which the interaction occurs were identified. All transfection experiments were performed at least in duplicate. The cells were harvested and lysed at $48 \mathrm{~h}$ post-transfection. The activities of the reporter firefly luciferase and the reference fluorescence Renilla luciferase were assayed using the dual- luciferase reporter assay system as instructed (Promega). Values were normalized to Renilla luciferase activities. Each experimental condition was measured in triplicate. Multiple transfections were performed for each constructed vector.

\section{Transient transfection and luciferase assays}

A Lipofectamine 2000 reagent (Invitrogen, Grand Island, NY) was used to multiply and transfect the same plasmid amount of constructed vectors into cells according to the designed ratio. Experimental condition and procedure of cell culture, transfection and luciferase assay are same as earlier described.

\section{RESULTS}

Two-hybrid system of restin and ATF3 indicating restin interaction with ATF3

A mammalian two-hybrid system effectively reflects the intracellular interaction between two proteins. In this system, the interaction of many intracellular proteins can be modified by glucosyl or phosphorylation, or be in a certain microenvironment. Using the mammalian twohybrid system to study the interaction between restin and ATF3 is advisable because of the phosphorylation in ATF3. A dual-luciferase reporter system was used in the present mammalian two-hybrid system. The two reporters Renilla and firefly luciferases were used to evaluate transfection efficiency and protein interaction intensity, respectively. In addition, Renilla luciferase was used as a control to normalize the results for comparison. Figure 1 revealed that ATF3 evidently interacted with restin. Similar results are obtained from the repeated experiments.

To further confirm the interaction of restin with ATF3 and determine the domain in which this interaction occurs, two two-hybrid plasmids were constructed. These plasmids contained the ATF3-activating domain (1 to 240 bp) and the DBD domain (241 to $543 \mathrm{bp}$ ), respectively, according to Genbank (Figure 2A). The primers for the activating domain were GCAGGATCCT GATGCTTCAACACCCAGG (sense) and TCGACGCGTCT CGGCTTTTGTGATG (anti-sense). The primers for the DBD domain were GCAGGATCGAGGTAGCCCCTGAA (sense) and TCGACGCGTGCTTAGCTCTGCAATGTTCC (anti-sense). After sequence confirmation, a twohybrid system was used to determine the domain that interacted with restin. The results reveal that restin interacted with the ATF3 DBD (Figure 2B). Using the restriction endonuclease Pst I site in the ATF3 DBD, ATF3 was cleaved into $\triangle A T F 3$ and $\triangle B D$ (Figure $2 A$ ) to identify the interaction of restin with ATF3 DBD. This cleavage was also performed to evaluate whether the interaction needed intact DBD. The results show that restin did not interact with the disintegrated DBD. This finding therefore proved that restin indeed interacted with ATF3, and that this interaction occurred in the ATF3 DBD (Figure 2B). 


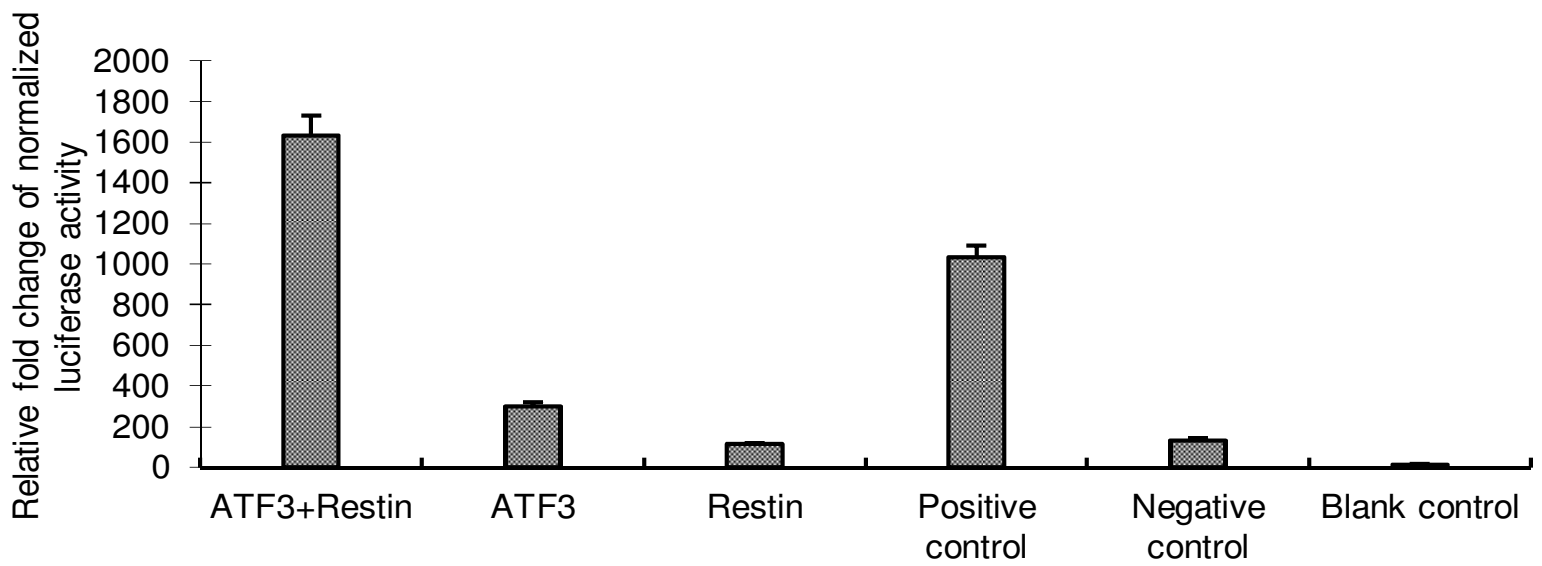

Figure 1. Screening of the interaction of restin with ATF3 by the two-hybrid system. The plasmids pACT-ATF3, pG5IUC, and pBIND-restin were transfected into COS-7. Reporter firefly luciferase activity was detected after $48 \mathrm{~h}$. The results show that restin intensively interacted with ATF3. The count unit used was light unit (LU). All the data were normalized at the same transfection efficiency. Data are the means of at least three experiments.

(A)

\begin{tabular}{|c|c|c|}
\hline $1 \mathrm{bp}$ & 240bp & \\
\hline ATF3 & $\perp$ & \\
\hline AD 1 bp & 240bp & \\
\hline & 241bp & $543 \mathrm{bp}$ \\
\hline$\triangle$ ATF3 1 bp & 345bp & \\
\hline$\triangle \mathrm{BD}$ & 345bp & 543bp \\
\hline
\end{tabular}

ATF3

546 bp

(B)

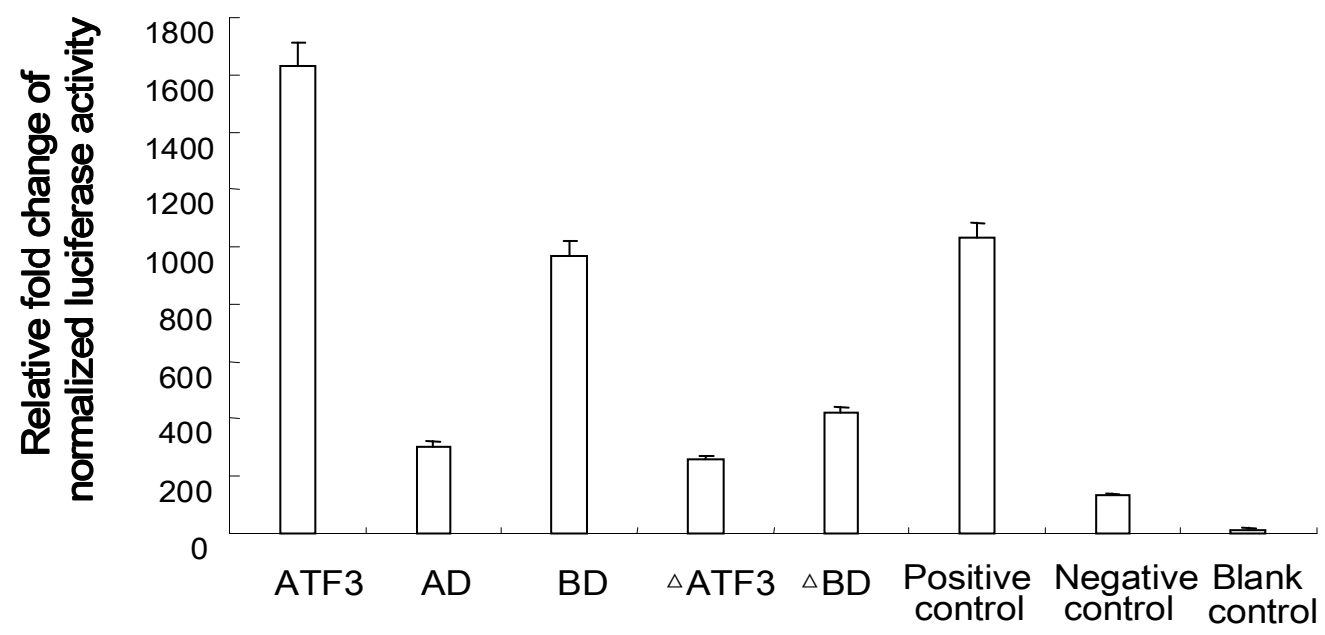

Figure 2. Schematic representation of the ATF3 functional domains and their interactions with restin. (A) Activating domain (AD, 1 to $240 \mathrm{bp}$ ); DNA binding domain (BD, 241 to $543 \mathrm{bp}$ ); $\triangle \mathrm{ATF} 3,1$ to $345 \mathrm{bp}$; $\triangle \mathrm{BD}, 346$ to $543 \mathrm{bp}$; and Pst I site, $345 \mathrm{bp}$. (B) Interaction of restin with the ATF3 functional domain and fragment. The result of the two-hybrid system show that restin interacts with the ATF3 DNA binding domain, and that this interaction is decreased after cleavage. Data are the means of at least three experiments. 
(A)

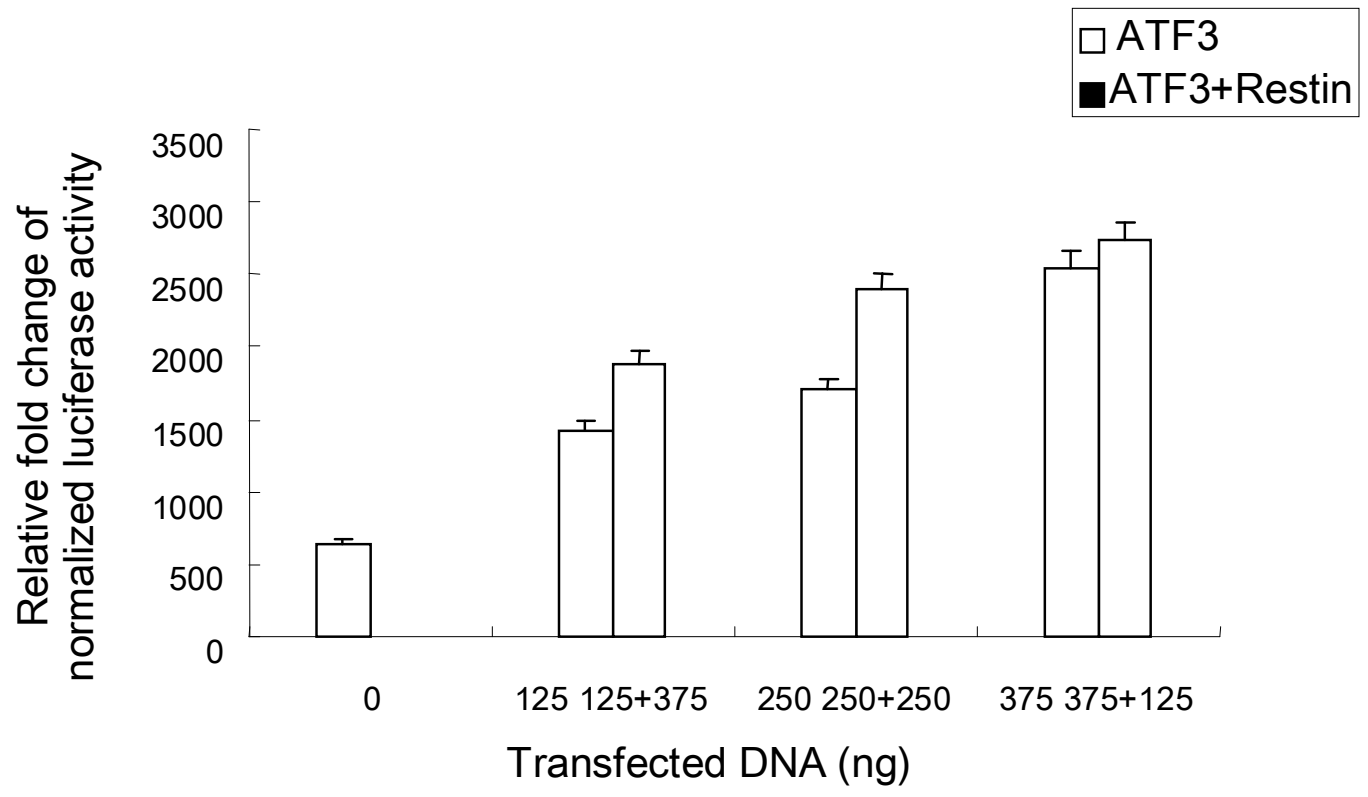

(B)

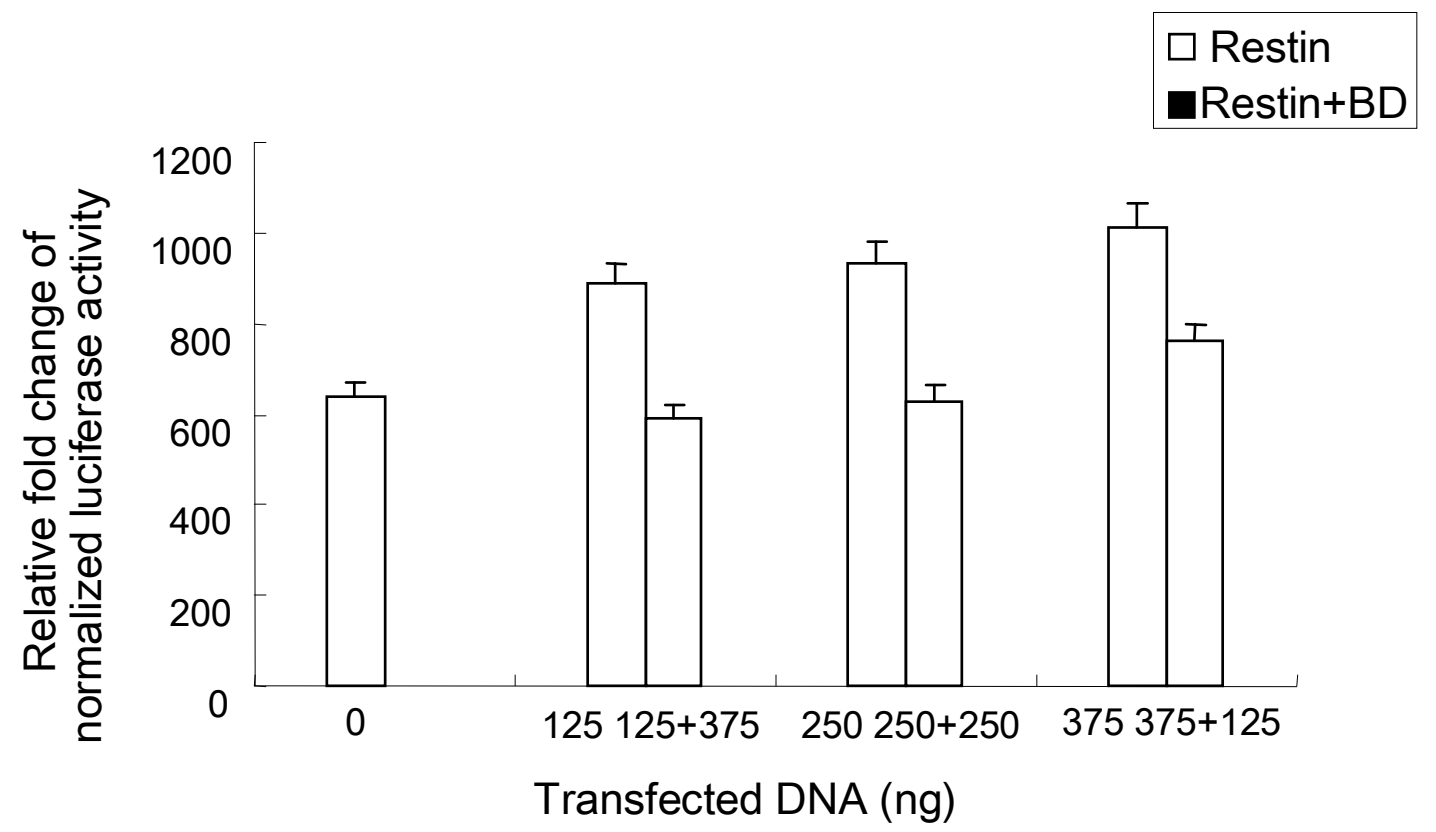

Figure 3. Assays of luciferase expression in NIH3T3 cells. Restin activates the transactivation of ATF3 (A), and this induction is mediated by the DNA binding domain of ATF3. (B) The cells were transfected with $1 \mu \mathrm{g} / \mathrm{well}$ research factor plasmids (pACT-ATF3, pACT-BD and pACT-restin) and empty vector pACT. The cells were also transfected with the same quantity of the reporter plasmid pATF/CRE-luc (300 ng/well) and the Renilla luciferase control vector pBind (50 ng/well).

\section{Restin activation of ATF3 transcription potentiated by the ATF3 DBD}

To clarify further the effect of restin on ATF3 activity, a luciferase expression reporter system for ATF3 was constructed. The results show that this activation was dose-dependent on the amount of transfected DNA (Figure $3 A$ ). In the experiments performed, the basal activity of ATF3 in the cells was low. This condition may reflect the lack of some important transcription factors for basal ATF3 transcription in the cells. Cotransfection with different ratios of restin and ATF3 into Hela and NIH3T3 
cells revealed that restin significantly affected ATF3 activity in a dose-dependent manner. The transfection of different doses of restin into Hela and NIH3T3 cells showed that restin weakly facilitated the basal activity of ATF3, and that the action among the three dose groups had no significant difference. However, when the ATF3 $\mathrm{BD}$ and restin were cotransfected into the cells, the $\mathrm{BD}$ intriguingly reversed the activation effect of restin. This phenomenon implied that the ATF3 BD mediated the activation effect of restin on ATF3.

\section{DISCUSSION}

The present study has demonstrated that restin at low concentrations activates the ATF3 promoter, and that this activation is mediated by the ATF3 BD. Restin, which is a non-CT MAGE like necdin and MAGE-D1, functions in cell proliferation inhibition (Francois et al., 2009; Maruyama et al., 1991; Reddy et al., 2010). As described earlier, ATF3 is a transcription factor initially expressed during cell stress responses. Many stimulating signals such as cytokine, ultraviolet radiation, injuries, etc. (Allan et al., 2001; James et al., 2006; Lu et al., 2006) could also induce the expression of ATF3. The expression of ATF3, which is often up-regulated in cancer (Ameri et al., 2007), has effects related to cellular protection. On one hand, ATF3 could induce cells to enter the cell cycle from the resting phase. For example, in a hepatocyte injury (Allan et al., 2001), ATF3 could induce the expression of cyclin D1 to induce hepatocyte entrance into the cell cycle. However, ATF3 overexpression inhibits cell proliferation. On the other hand, ATF3 as a functional homodimer may repress transcription. In the study of Chen et al. (1996), ATF3 represses rather than activates transcription from promoters with ATF sites. The transcription is repressed by stabilizing inhibitory cofactors at the promoter site.

In the present study, restin has been found to intensively interact with ATF3. The cell proliferation inhibition activity of restin possibly correlates with ATF3. We have suggested that alkali MAGEs may actually be inhibitive factors that maintain cell silence and inhibit different signal pathways. Alkali MAGEs are highly expressed in differentiated terminal cells, and are indicators of cell proliferation inhibition. Necdin, for example inhibits the activity of the transcription factors E2F and p53, which act in the major regulation pathway of cell proliferation. Our results herein indicate that the binding of restin to the ATF3 BD has resulted in an up-regulation of ATF3 transcription. Hence, the interaction of restin with ATF3 possibly activates the transcriptional activity of ATF3. Consequently, ATF3 could not start the cell cycle as homodimers with other cell factors. The results have also shown that restin functions by binding to the consensus domain of ATF3, in agreement with a previous study (Stearns et al., 2004).
However, these results appear to contradict other data showing that ATF3 could induce cells to enter the cell cycle from the resting phase. We suspect that the effect of restin on ATF3 in terms of suppressing cell proliferation is dependent on the secondary action of ATF3. This secondary action refers to the formation of homodimers and selective heterodimers with certain other bZip proteins (Ageez et al., 2003; Sakakibara et al., 2001; Suckow et al., 1993). This is only a speculation at this point, and the model proposed herein requires further investigation. More work is therefore needed to determine whether the effect of restin on ATF3 is tissue-specific, stress-specific, and/or cell cycle modulation-specific. Further investigation is also needed to identify the components of these regulatory complexes, as well as their effects on various cell conditions and cell types.

\section{ACKNOWLEDGEMENTS}

The authors gratefully acknowledge Drs. Zhongliang Zhao, Qin Pu and Fan Lu for the plasmids. Our colleagues in the Department of Biochemistry of the Fourth Military Medical University are also acknowledged for contributing ideas and suggestions to the study over the years.

\section{REFERENCES}

Ageez A, Matsunaga S, Uchida W, Sugiyama R, Kazama Y, Kawano S (2003). Isolation and characterization of two homeodomain leucine zipper genes from the dioecious plant Silene latifolia. Genes. Genet. Syst. 78(5): 353-361.

Allan AL, Albanese C, Pestell RG, Lamarre J (2001). Activating transcription factor 3 induces DNA synthesis and expression of cyclin D1 in hepatocytes. J. Biol. Chem. 276(29): 27272-27280.

Ameri K, Hammond EM, Culmsee C, Raida M, Katschinski DM, Wenger RH, Wagner E, Davis RJ, Hai T, Denko N, Harris AL (2007). Induction of activating transcription factor 3 by anoxia is independent of p53 and the hypoxic HIF signalling pathway. Oncogene, 26(2): 284-289.

Aprelikova O, Pandolfi S, Tackett S, Ferreira M, Salnikow K, Ward Y, Risinger JI, Barrett JC, Niederhuber J (2009). Melanoma antigen-11 inhibits the hypoxia-inducible factor prolyl hydroxylase 2 and activates hypoxic response. Cancer Res. 69(2): 616-624.

Chen BP, Wolfgang CD, Hai T (1996). Analysis of ATF3, a transcription factor induced by physiological stresses and modulated by gadd153/Chop10. Mol. Cell. Biol. 16(3): 1157-1168.

Chomez P, De Backer O, Bertrand M, De Plaen E, Boon T, Lucas S (2001). An overview of the MAGE gene family with the identification of all human members of the family. Cancer Res. 61(14): 5544-5551.

Francois V, Ottaviani S, Renkvist N, Stockis J, Schuler G, Thielemans $\mathrm{K}$, Colau D, Marchand M, Boon T, Lucas S, Van Der Bruggen $\mathrm{P}$ (2009). The CD4(+) T-cell response of melanoma patients to a MAGE-A3 peptide vaccine involves potential regulatory $\mathrm{T}$ cells. Cancer Res. 69(10): 4335-4345.

Freshney R (1994). Culture of animal cells:a menu of basic techniques. Wiley-liss. Inc. New York.

James CG, Woods A, Underhill TM, Beier F (2006). The transcription factor ATF3 is upregulated during chondrocyte differentiation and represses cyclin D1 and A gene transcription. BMC Mol. Biol. 7: 30.

Kirkin AF, Dzhandzhugazyan K, Zeuthen J (1998). Melanomaassociated antigens recognized by cytotoxic $\mathrm{T}$ lymphocytes. Apmis, 106(7): 665-679. 
Lu D, Wolfgang CD, Hai T (2006). Activating transcription factor 3, a stress-inducible gene, suppresses Ras-stimulated tumorigenesis. J. Biol. Chem. 281(15): 10473-10481.

Lucas S, De Smet C, Arden KC, Viars CS, Lethe B, Lurquin C, Boon T (1998). Identification of a new MAGE gene with tumor-specific expression by representational difference analysis. Cancer Res. 58(4): 743-752.

Maruyama K, Usami M, Aizawa T, Yoshikawa K (1991). A novel brainspecific mRNA encoding nuclear protein (necdin) expressed in neurally differentiated embryonal carcinoma cells. Biochem. Biophys. Res. Commun. 178(1): 291-296.

Reddy EM, Chettiar ST, Kaur N, Shepal V, Shiras A (2010). DIxin-1, a MAGE family protein, induces accelerated neurite outgrowth and cell survival by enhanced and early activation of MEK and Akt signalling pathways in PC12 cells. Exp. Cell. Res. 316(14): 2220-2236.

Sakakibara K, Nishiyama T, Kato M, Hasebe M (2001). Isolation of homeodomain-leucine zipper genes from the moss Physcomitrella patens and the evolution of homeodomain-leucine zipper genes in land plants. Mol. Biol. Evol. 18(4): 491-502.

Stearns ME, Kim G, Garcia F, Wang M (2004). Interleukin-10 induced activating transcription factor 3 transcriptional suppression of matrix metalloproteinase-2 gene expression in human prostate CPTX-1532 Cells. Mol. Cancer Res. 2(7): 403-416.

Suckow M, Von Wilcken-Bergmann B, Muller-Hill B (1993). Identification of three residues in the basic regions of the bZIP proteins GCN4, $\mathrm{C} / \mathrm{EBP}$ and TAF-1 that are involved in specific DNA binding. Embo. J. 12(3): 1193-1200.
Wu Y, Lu F, Qi Y, Wang R, Zhang J, Lu Z, Zhao Z (2005). Interaction of restin with transcription factors. Sci. China. C. Life. Sci. 48(3): 256262.

Xu JQ, Deng JL, Wu YS, Fu HY, Wang RH, Zhang J, Lu F, Zhao ZL (2006). Construction and activity assay of the activating transcription factor 3 reporter vector pATF/CRE-luc. Acta. Biochim. Biophys. Sin (Shanghai). 38(1): 58-62.

Zhao Z, Lu F, Zhu F, Yang H, Chai Y, Chen S (2002). Cloning and biological comparison of Restin, novel member of Mage superfamily. Sci. China. C. Life. Sci. 45(4): 412-420.

Zhu F, Yan W, Zhao ZL, Chai YB, Lu F, Wang Q, Peng WD, Yang AG, Wang CJ (2000). Improved PCR-based subtractive hybridization strategy for cloning differentially expressed genes. Biotechniques, 29(2): 310-313. 